\title{
Certain Subclasses of $p$-Valent Meromorphic Functions Associated with a New Operator
}

\author{
A. O. Mostafa, M. K. Aouf, A. Shamandy, and E. A. Adwan \\ Department of Mathematics, Faculty of Science, Mansoura University, Mansoura 35516, Egypt \\ Correspondence should be addressed to E. A. Adwan; eman.a2009@yahoo.com \\ Received 21 December 2012; Accepted 10 March 2013 \\ Academic Editor: J. Dziok
}

Copyright (c) 2013 A. O. Mostafa et al. This is an open access article distributed under the Creative Commons Attribution License, which permits unrestricted use, distribution, and reproduction in any medium, provided the original work is properly cited.

We introduce two classes of $p$-valent meromorphic functions associated with a new operator and derive several interesting results for these classes.

\section{Introduction}

Let $\Sigma_{p}$ denote the class of functions of the form

$$
f(z)=z^{-p}+\sum_{n=1}^{\infty} a_{n-p} z^{n-p} \quad(p \in \mathbb{N}=\{1,2,3, \ldots\}),
$$

which are analytic and $p$-valent in the punctured unit disc $U^{*}=\{z \in \mathbb{C}: 0<|z|<1\}=U \backslash\{0\}$. Let $P_{k}(\beta)$ be the class of functions $\phi(z)$ analytic in $U$ satisfying the properties $h(0)=1$ and

$$
\int_{0}^{2 \pi}\left|\frac{\Re\{h(z)\}-\beta}{1-\beta}\right| d \theta \leq k \pi
$$

where $z=r e^{i \theta}, k \geq 2$, and $0 \leq \beta<1$. The class $P_{k}(\beta)$ was introduced by Padmanabhan and Parvatham [1]. For $\beta=0$, the class $P_{k}(0)=P_{k}$ was introduced by Pinchuk [2]. Also we note that $P_{2}(\beta)=P(\beta)$, where $P(\beta)$ is the class of functions with positive real part greater than $\beta$ and $P_{2}(0)=P$, where $P$ is the class of functions with positive real part. From (2), we have $h(z) \in P_{k}(\beta)$ if and only if there exists $h_{1}, h_{2} \in P(\beta)$ such that

$$
h(z)=\left(\frac{k}{4}+\frac{1}{2}\right) h_{1}(z)-\left(\frac{k}{4}-\frac{1}{2}\right) h_{2}(z) \quad(z \in U) .
$$
by

For functions $f(z) \in \Sigma_{p}$ given by (1) and $g(z) \in \Sigma_{p}$ given

$$
g(z)=z^{-p}+\sum_{n=1}^{\infty} a_{n-p} z^{n-p} \quad(p \in \mathbb{N})
$$

the Hadamard product (or convolution) of $f(z)$ and $g(z)$ is defined by

$$
(f * g)(z)=z^{-p}+\sum_{n=1}^{\infty} a_{n-p} b_{n-p} z^{n-p}=(g * f)(z) .
$$

Aqlan et al. [4] defined the operator $Q_{\beta, p}^{\alpha}: \Sigma_{p} \rightarrow \Sigma_{p}$ by

$$
Q_{\beta, p}^{\alpha} f(z)=\left\{\begin{array}{c}
z^{-p}+\frac{\Gamma(\alpha+\beta)}{\Gamma(\beta)} \sum_{n=1}^{\infty} \frac{\Gamma(n+\beta)}{\Gamma(n+\beta+\alpha)} a_{n-p} z^{n-p} \\
\left(\alpha>0 ; \beta>-1 ; p \in \mathbb{N} ; f \in \Sigma_{p}\right) \\
f(z) \quad\left(\alpha=0 ; \beta>-1 ; p \in \mathbb{N} ; f \in \Sigma_{p}\right) .
\end{array}\right.
$$

Mostafa [5] used Aqlan et al. operator and defined the following linear operator $H_{p, \beta, \mu}^{\alpha}: \Sigma_{p} \rightarrow \Sigma_{p}$ as follows.

First put

$$
G_{\beta, p}^{\alpha}(z)=z^{-p}+\frac{\Gamma(\alpha+\beta)}{\Gamma(\beta)} \sum_{n=1}^{\infty} \frac{\Gamma(n+\beta)}{\Gamma(n+\beta+\alpha)} z^{n-p} \quad(p \in \mathbb{N})
$$

It is known that the class $P_{k}(\beta)$ is a convex set (see [3]). 
and let $G_{\beta, p, \mu}^{\alpha *}$ be defined by

$$
G_{\beta, p}^{\alpha}(z) * G_{\beta, p, \mu}^{\alpha *}(z)=\frac{1}{z^{p}(1-z)^{\mu}} \quad(\mu>0 ; p \in \mathbb{N}) .
$$

Then

$$
H_{p, \beta, \mu}^{\alpha} f(z)=G_{\beta, p}^{\alpha *}(z) * f(z) \quad\left(f \in \Sigma_{p}\right) .
$$

Using (7) and (9), we have

$$
\begin{aligned}
& H_{p, \beta, \mu}^{\alpha} f(z) \\
& \quad=z^{-p}+\frac{\Gamma(\beta)}{\Gamma(\alpha+\beta)} \sum_{n=1}^{\infty} \frac{\Gamma(n+\beta+\alpha)(\mu)_{n}}{\Gamma(n+\beta)(1)_{n}} a_{n-p} z^{n-p},
\end{aligned}
$$

where $(\nu)_{n}$ denotes the Pochhammer symbol given by

$$
\begin{aligned}
(\nu)_{n} & =\frac{\Gamma(\nu+n)}{\Gamma(\nu)} \\
& = \begin{cases}1 & (n=0) \\
\nu(\nu+1) \cdots(\nu+n-1) & (n \in \mathbb{N}) .\end{cases}
\end{aligned}
$$

It is readily verified from (10) that (see [5])

$$
\begin{aligned}
z\left(H_{p, \beta, \mu}^{\alpha} f(z)\right)^{\prime}= & (\alpha+\beta) H_{p, \beta, \mu}^{\alpha+1} f(z) \\
& -(\alpha+\beta+p) H_{p, \beta, \mu}^{\alpha} f(z), \\
z\left(H_{p, \beta, \mu}^{\alpha} f(z)\right)^{\prime}= & \mu H_{p, \beta, \mu+1}^{\alpha} f(z) \\
& -(\mu+p) H_{p, \beta, \mu}^{\alpha} f(z) .
\end{aligned}
$$

It is noticed that by putting $\mu=1$ in (10), we obtain the operator

$$
\begin{aligned}
H_{p, \beta, 1}^{\alpha} f(z) & =H_{p, \beta}^{\alpha} f(z) \\
& =z^{-p}+\frac{\Gamma(\beta)}{\Gamma(\alpha+\beta)} \sum_{n=1}^{\infty} \frac{\Gamma(n+\alpha+\beta)}{\Gamma(n+\beta)} a_{n-p} z^{n-p}
\end{aligned}
$$

Now, by using the linear operator $H_{p, \beta, \mu}^{\alpha} f(z)$, we introduce classes of $p$-valent Bazilevic functions of $\Sigma_{p}$ as follows.

Definition 1. A function $f(z) \in \Sigma_{p}$ is said to be in the class $K_{p}(\alpha, \beta, \mu, \delta, \rho, \gamma)$ if it satisfies the following condition:

$$
\begin{aligned}
& {\left[(1-\gamma)\left(z^{p} H_{p, \beta, \mu}^{\alpha} f(z)\right)^{\delta}\right.} \\
& \left.+\gamma\left(\frac{H_{p, \beta, \mu}^{\alpha+1} f(z) f(z)}{H_{p, \beta, \mu}^{\alpha} f(z)}\right)\left(z^{p} H_{p, \beta, \mu}^{\alpha} f(z)\right)^{\delta}\right] \in \mathscr{P}_{k}(\rho) \\
& (\alpha, \mu>0 ; \beta>-1 ; k \geq 2 ; \gamma \geq 0, \\
& \delta>0 ; 0 \leq \rho<1 ; p \in \mathbb{N} ; z \in U) .
\end{aligned}
$$

Definition 2. A function $f(z) \in \Sigma_{p}$ is said to be in the class $M_{p}(\alpha, \beta, \mu, \delta, \rho, \gamma)$ if it satisfies the following condition:

$$
\begin{aligned}
& {\left[(1-\gamma)\left(z^{p} H_{p, \beta, \mu}^{\alpha} f(z)\right)^{\delta}\right.} \\
& \left.+\gamma\left(\frac{H_{p, \beta, \mu+1}^{\alpha} f(z) f(z)}{H_{p, \beta, \mu}^{\alpha} f(z)}\right)\left(z^{p} H_{p, \beta, \mu}^{\alpha} f(z)\right)^{\delta}\right] \in \mathscr{P}_{k}(\rho) \\
& (\alpha, \mu>0 ; \beta>-1 ; k \geq 2 ; \gamma \geq 0, \\
& \delta>0 ; 0 \leq \rho<1 ; p \in \mathbb{N} ; z \in U) .
\end{aligned}
$$

In this paper, we investigate several properties of the classes $K_{p}(\alpha, \beta, \mu, \delta, \rho, \gamma)$ and $M_{p}(\alpha, \beta, \mu, \delta, \rho, \gamma)$.

\section{Main Results}

Unless otherwise mentioned, we assume throughout this paper that $k \geq 2, \alpha, \delta, \gamma, \mu>0, \beta>-1, \alpha+\beta>0,0 \leq \rho<1$, $p \in \mathbb{N}$ and all powers are understood as principle values.

To prove our results we need the following lemma.

Lemma 3 (see [6]). Let $u=u_{1}+i u_{2}, v=v_{1}+i v_{2}$ and $\Phi(u, v)$ be a complex-valued function satisfying the conditions:

(i) $\Phi(u, v)$ is continuous in a domain $D \in \mathbb{C}^{2}$.

(ii) $(0,1) \in D$ and $\Phi(1,0)>0$.

(iii) $\mathfrak{R}\left\{\Phi\left(i u_{2}, v_{1}\right)\right\}>0$ whenever $\left(i u_{2}, v_{1}\right) \in D$ and $v_{1} \leq$ $-(1 / 2)\left(1+u_{2}^{2}\right)$.

If $h(z)=1+c_{n} z^{n}+c_{n+1} z^{n+1}+\cdots$ is analytic in $U$ such that $\left(h(z), z h^{\prime}(z)\right) \in D$ and $\mathfrak{R}\left\{\Phi\left(h(z), z h^{\prime}(z)\right)\right\}>0$ for $z \in U$, then $\Re\{h(z)\}>0$ in $U$.

Employing the technique used by Noor and Muhammad [7] and Aouf and Seoudy [8] for multivalent functions, we prove the following theorems.

Theorem 4. If $f(z) \in K_{p}(\alpha, \beta, \mu, \delta, \rho, \gamma)$, then

$$
\left(z^{p} H_{p, \beta, \mu}^{\alpha} f(z)\right)^{\delta} \in \mathscr{P}_{k}(\eta),
$$

where $\eta$ is given by

$$
\eta=\frac{2 \delta \rho(\alpha+\beta)+\gamma}{2 \delta(\alpha+\beta)+\gamma} .
$$

Proof. Setting

$$
\begin{aligned}
&\left(z^{p} H_{p, \beta, \mu}^{\alpha} f(z)\right)^{\delta} \\
&= H(z)=(1-\eta) h(z)+\eta \\
&=\left(\frac{k}{4}+\frac{1}{2}\right)\left\{(1-\eta) h_{1}(z)+\eta\right\} \\
&-\left(\frac{k}{4}-\frac{1}{2}\right)\left\{(1-\eta) h_{2}(z)+\eta\right\},
\end{aligned}
$$


where $h_{i}(z)(i=1,2)$ are analytic in $U$ with $h_{i}(0)=1(i=$ $1,2)$, and $h(z)$ is given by (3). Differentiating both sides of (19) with respect to $z$ and using (12) in the resulting equation, we obtain

$$
\begin{aligned}
& {\left[(1-\gamma)\left(z^{p} H_{p, \beta, \mu}^{\alpha} f(z)\right)^{\delta}\right.} \\
& \left.+\gamma\left(\frac{H_{p, \beta, \mu}^{\alpha+1} f(z) f(z)}{H_{p, \beta, \mu}^{\alpha} f(z)}\right)\left(z^{p} H_{p, \beta, \mu}^{\alpha} f(z)\right)^{\delta}\right] \\
& =\{(1-\eta) h(z) \\
& \left.\quad+\eta+\frac{\gamma(1-\eta) z h^{\prime}(z)}{\delta(\alpha+\beta)}\right\} \in P_{k}(\rho) \quad(z \in U),
\end{aligned}
$$

which implies that

$$
\begin{aligned}
& \frac{1}{1-\rho}\left\{\eta-\rho+(1-\eta) h_{i}(z)\right. \\
& \left.\quad+\frac{\gamma(1-\eta) z h_{i}^{\prime}(z)}{\delta(\alpha+\beta)}\right\} \in P \quad(z \in U ; i=1,2) .
\end{aligned}
$$

We form the functional $\Phi(u, v)$ by choosing $u=h_{i}(z), v=$ $z h_{i}^{\prime}(z)$, that is,

$$
\Phi(u, v)=\eta-\rho+(1-\eta) u+\frac{\gamma(1-\eta) v}{\delta(\alpha+\beta)} .
$$

Clearly, the first two conditions of Lemma 3 are satisfied. Now, we verify the condition (iii) as follows:

$$
\begin{aligned}
\Re\left\{\Phi\left(i u_{2}, v_{1}\right)\right\} & =\eta-\rho+\mathfrak{R}\left\{\frac{\gamma(1-\eta) v_{1}}{\delta(\alpha+\beta)}\right\} \\
& \leq \eta-\rho-\frac{\gamma(1-\eta)\left(1+u_{2}^{2}\right)}{2 \delta(\alpha+\beta)} \\
& =\frac{A+B u_{2}^{2}}{2 C},
\end{aligned}
$$

where

$$
\begin{gathered}
A=2 \delta(\alpha+\beta)(\eta-\rho)-\gamma(1-\eta), \\
B=-\gamma(1-\eta), \\
C=2 \delta(\alpha+\beta) .
\end{gathered}
$$

We note that $\mathfrak{R}\left\{\Phi\left(i u_{2}, v_{1}\right)\right\}<0$ if and only if $A=0, B<0$. From $\eta$ given by (18), we have $0 \leq \eta<1, A=0$, and $B<0$. Therefore, applying Lemma 3 , we have $h_{i}(z) \in P(i=1,2)$ and consequently $H(z) \in P_{k}(\eta)$ for $z \in U$. This completes the proof of Theorem 4 .

Similarly, we can prove the following theorem for the class $M_{p}(\alpha, \beta, \mu, \delta, \rho, \gamma)$.
Theorem 5. If $f(z) \in M_{p}(\alpha, \beta, \mu, \delta, \rho, \gamma)$, then

$$
\left(z^{p} H_{p, \beta, \mu}^{\alpha} f(z)\right)^{\delta} \in \mathscr{P}_{k}(\chi),
$$

where $\chi$ is given by

$$
\chi=\frac{2 \delta \mu \rho+\gamma}{2 \delta \mu+\gamma} .
$$

Theorem 6. If $f(z) \in K_{p}(\alpha, \beta, \mu, \delta, \rho, \gamma)$, then

$$
\left(z^{p} H_{p, \beta, \mu}^{\alpha} f(z)\right)^{\delta / 2} \in \mathscr{P}_{k}(\zeta),
$$

where $\zeta$ is given by

$$
\zeta=\frac{\gamma+\sqrt{\gamma^{2}+4 \rho \delta(\alpha+\beta)[\delta(\alpha+\beta)+\gamma]}}{2[\delta(\alpha+\beta)+\gamma]} .
$$

Proof. Let $f(z) \in K_{p}(\alpha, \beta, \mu, \delta, \rho, \gamma)$ and

$$
\begin{aligned}
\left(z^{p} H_{p, \beta, \mu}^{\alpha} f(z)\right)^{\delta}= & M(z)=[(1-\zeta) h(z)+\zeta]^{2} \\
= & \left(\frac{k}{4}+\frac{1}{2}\right)\left[(1-\zeta) h_{1}(z)+\zeta\right]^{2} \\
& -\left(\frac{k}{4}-\frac{1}{2}\right)\left[(1-\zeta) h_{2}(z)+\zeta\right]^{2},
\end{aligned}
$$

where $h_{i}(z)(i=1,2)$ are analytic in $U$ with $h_{i}(0)=1(i=$ $1,2)$ and $h(z)$ is given by (3). Differentiating both sides of (29) with respect to $z$ and using (12) in the resulting equation, we obtain

$$
\begin{aligned}
& {\left[(1-\gamma)\left(z^{p} H_{p, \beta, \mu}^{\alpha} f(z)\right)^{\delta}\right.} \\
& \left.\quad+\gamma\left(\frac{H_{p, \beta, \mu}^{\alpha+1} f(z) f(z)}{H_{p, \beta, \mu}^{\alpha} f(z)}\right)\left(z^{p} H_{p, \beta, \mu}^{\alpha} f(z)\right)^{\delta}\right] \\
& =\left\{[(1-\zeta) h(z)+\zeta]^{2}+[(1-\zeta) h(z)+\zeta]\right. \\
& \left.\quad \times \frac{2 \gamma(1-\zeta) z h^{\prime}(z)}{\delta(\alpha+\beta)}\right\} \in P_{k}(\rho) \quad(z \in U),
\end{aligned}
$$

which implies that

$$
\begin{aligned}
\frac{1}{1-\rho}\{ & {[(1-\zeta) h(z)+\zeta]^{2}+[(1-\zeta) h(z)+\zeta] } \\
& \left.\times \frac{2 \gamma(1-\zeta) z h^{\prime}(z)}{\delta(\alpha+\beta)}-\rho\right\} \in P \quad(i=1,2) .
\end{aligned}
$$

We form the functional $\Phi(u, v)$ by choosing $u=h_{i}(z), v=$ $z h_{i}^{\prime}(z)$, that is,

$$
\Phi(u, v)=[(1-\zeta) u+\zeta]^{2}+[(1-\zeta) u+\zeta] \frac{2 \gamma(1-\zeta) v}{\delta(\alpha+\beta)}-\rho
$$


Clearly, the conditions (i) and (ii) of Lemma 3 are satisfied. Now, we verify the condition (iii) as follows:

$$
\begin{aligned}
\mathfrak{R}\left\{\Phi\left(i u_{2}, v_{1}\right)\right\} & =\zeta^{2}-(1-\zeta)^{2} u_{2}^{2}+\frac{2 \gamma(1-\zeta) v_{1}}{\delta(\alpha+\beta)}-\rho \\
& \leq \zeta^{2}-\rho-(1-\zeta)^{2} u_{2}^{2}-\frac{\gamma \zeta(1-\zeta)\left(1+u_{2}^{2}\right)}{\delta(\alpha+\beta)} \\
& =\frac{A+B u_{2}^{2}}{2 C},
\end{aligned}
$$

where

$$
\begin{gathered}
A=\zeta^{2} \delta(\alpha+\beta)-\gamma \zeta(1-\zeta)-\rho \delta(\alpha+\beta), \\
B=-\left[(1-\zeta)^{2}+\gamma \zeta(1-\zeta)\right], \\
C=\frac{\delta(\alpha+\beta)}{2} .
\end{gathered}
$$

We note that $\mathfrak{R}\left\{\Phi\left(i u_{2}, v_{1}\right)\right\}<0$ if and only if $A=0, B<0$. From $\zeta$ as given by (28), we have $0 \leq \zeta<1, A=0$, and $B<0$. Therefore, applying Lemma 3 , we have $h_{i}(z) \in P(i=1,2)$ and consequently $M(z) \in P_{k}(\zeta)$ for $z \in U$. This completes the proof of Theorem 6 .

Similarly, we can prove the following theorem for the class $M_{p}(\alpha, \beta, \mu, \delta, \rho, \gamma)$.

Theorem 7. If $f(z) \in M_{p}(\alpha, \beta, \mu, \delta, \rho, \gamma)$, then

$$
\left(z^{p} H_{p, \beta, \mu}^{\alpha} f(z)\right)^{\delta / 2} \in \mathscr{P}_{k}(\lambda)
$$

where $\lambda$ is given by

$$
\lambda=\frac{\gamma+\sqrt{\gamma^{2}+\rho \delta \mu(2 \gamma+\delta \mu)}}{(2 \gamma+\delta \mu)} .
$$

Remark 8. Putting $\mu=1$, in Theorems 4 and 6, we obtain the corresponding results for the operator $H_{p, \beta}^{\alpha}$ defined in (14).

\section{Acknowledgment}

The authors would like to thank the referees of the paper for their helpful suggestions.

\section{References}

[1] K. S. Padmanabhan and R. Parvatham, "Properties of a class of functions with bounded boundary rotation," Annales Polonici Mathematici, vol. 31, no. 3, pp. 311-323, 1975/76.

[2] B. Pinchuk, "Functions of bounded boundary rotation," Israel Journal of Mathematics, vol. 10, pp. 6-16, 1971.

[3] K. I. Noor, "On subclasses of close-to-convex functions of higher order," International Journal of Mathematics and Mathematical Sciences, vol. 15, no. 2, pp. 279-289, 1992.
[4] E. Aqlan, J. M. Jahangiri, and S. R. Kulkarni, "Certain integral operators applied to meromorphic $p$-valent functions," Journal of Natural Geometry, vol. 24, no. 1-2, pp. 111-120, 2003.

[5] A. Mostafa, "Inclusion results for certain subclasses of $p$ valent meromorphic functions associated with a new operator," Journal of Inequalities and Applications, vol. 2012, p. 169, 2012.

[6] S. S. Miller and P. T. Mocanu, "Second-order differential inequalities in the complex plane," Journal of Mathematical Analysis and Applications, vol. 65, no. 2, pp. 289-305, 1978.

[7] K. I. Noor and A. Muhammad, "Some properties of the subclass of $p$-valent Bazilevic functions," Acta Universitatis Apulensis, no. 17, pp. 189-197, 2009.

[8] M. K. Aouf and T. M. Seoudy, "Some properties of certain subclasses of $p$-valent Bazilevic functions associated with the generalized operator," Applied Mathematics Letters, vol. 24, no. 11, pp. 1953-1958, 2011. 


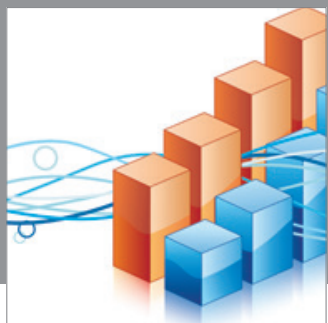

Advances in

Operations Research

mansans

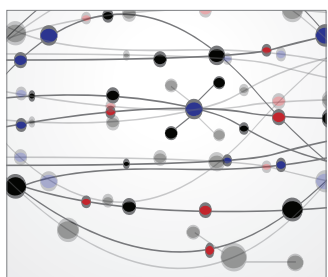

The Scientific World Journal
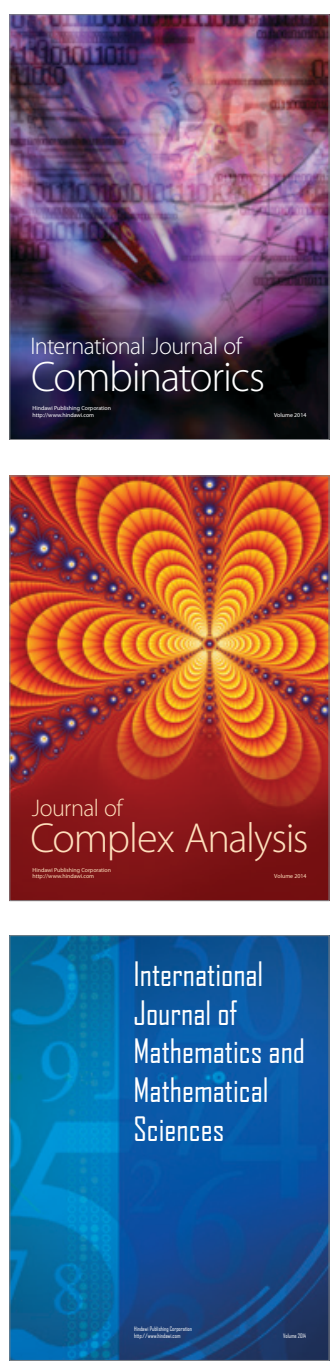
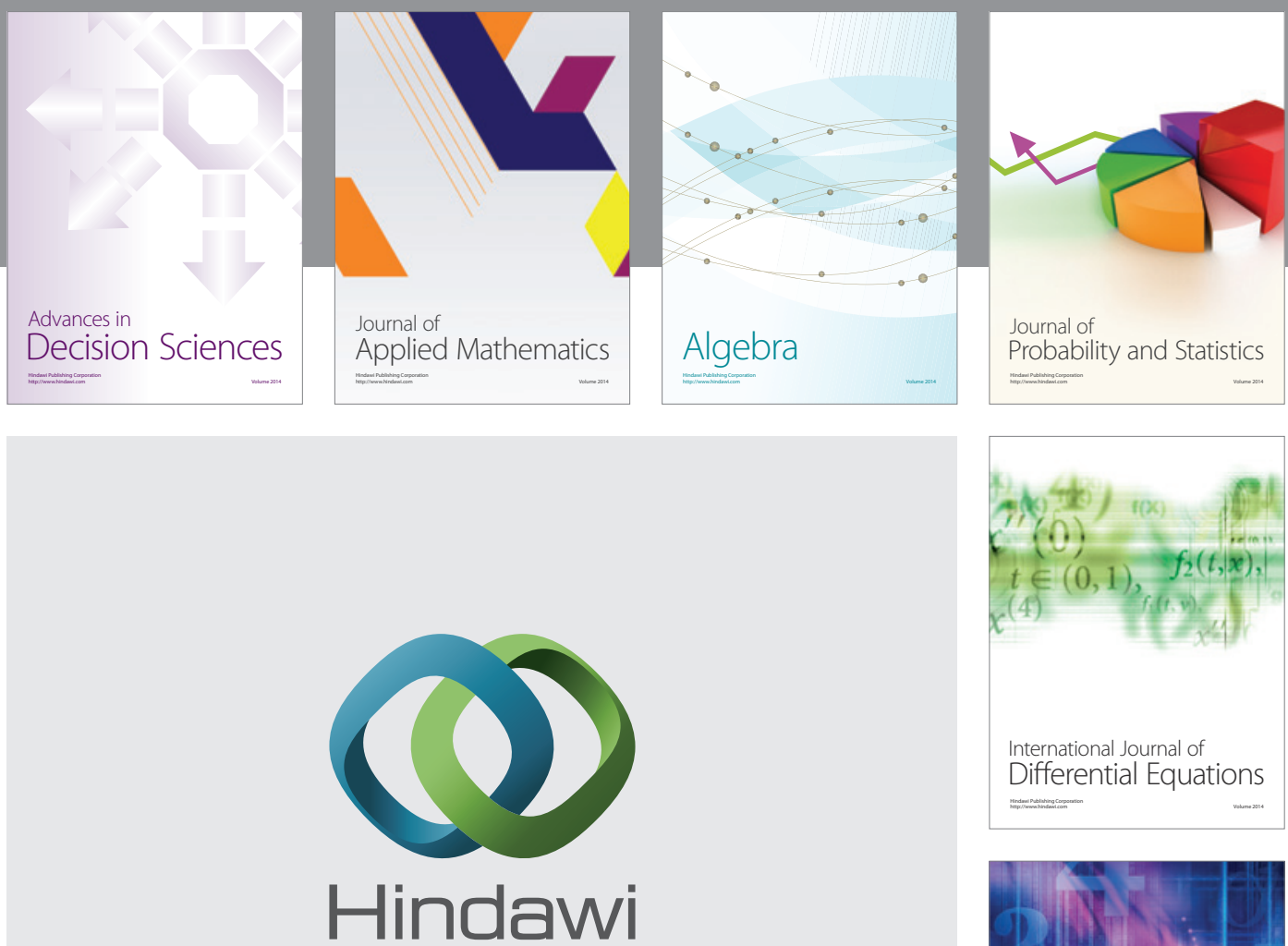

Submit your manuscripts at http://www.hindawi.com
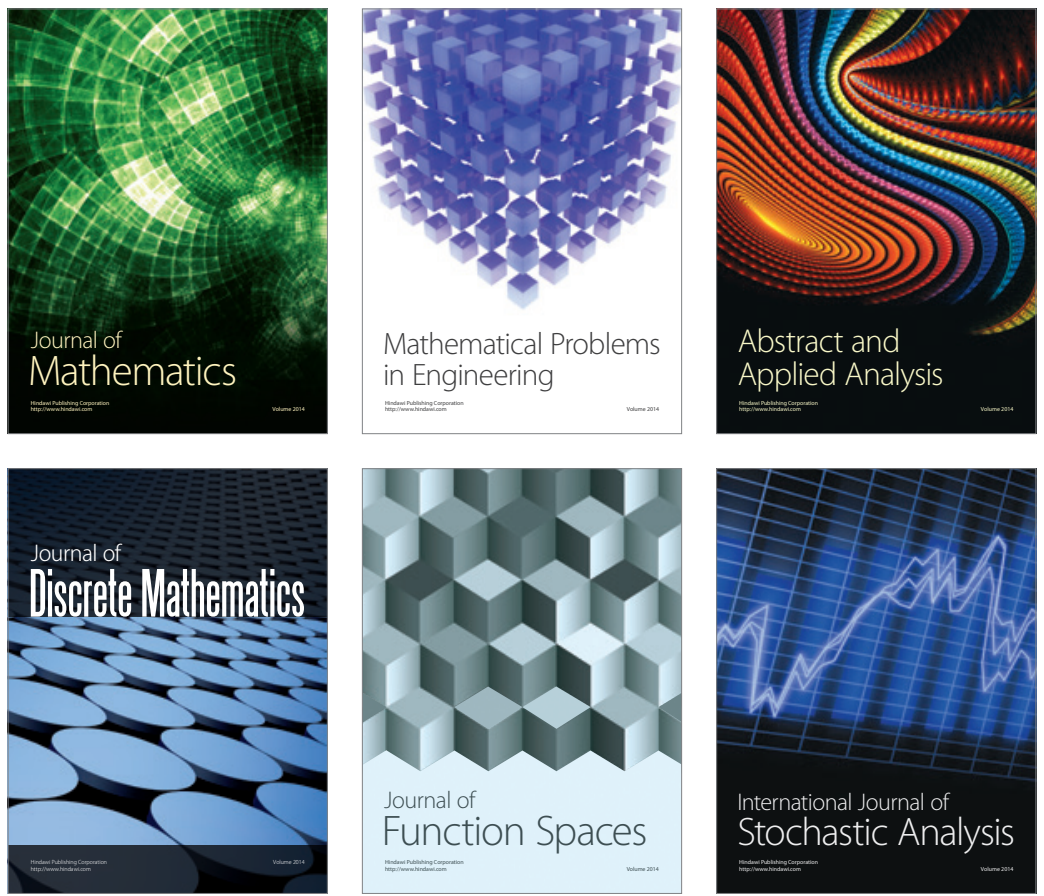

Journal of

Function Spaces

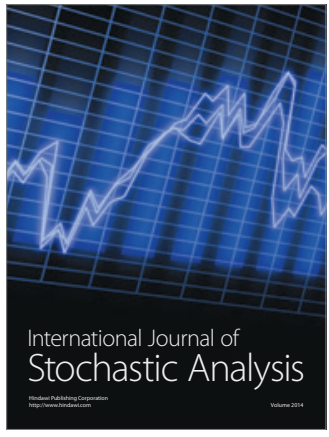

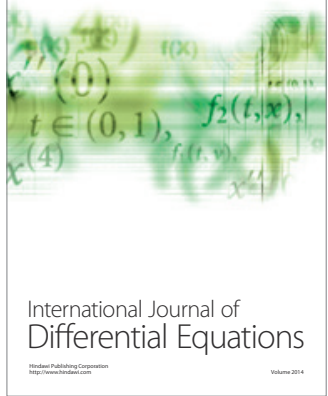
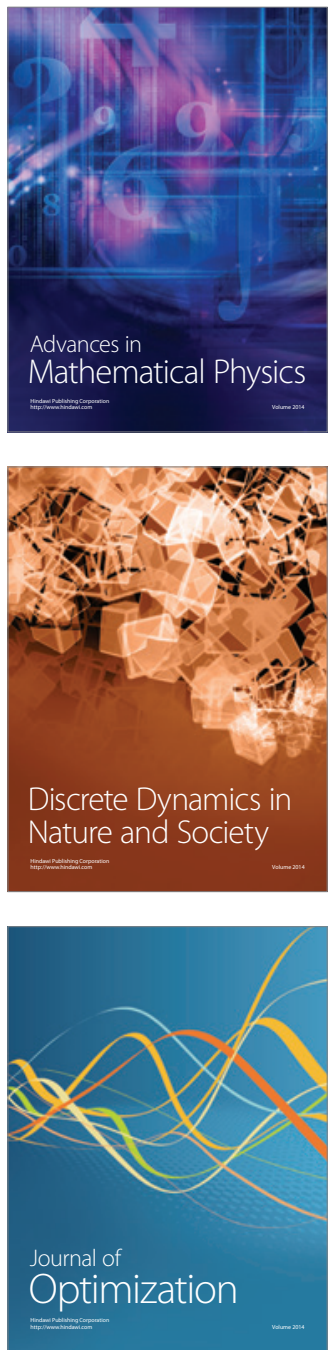